\title{
Fence Placement for Legacy Data-Race-Free Programs via Synchronization Read Detection
}

\author{
ANDREW J. MCPHERSON and VIJAY NAGARAJAN, University of Edinburgh \\ SUSMIT SARKAR, University of St. Andrews \\ MARCELO CINTRA, Intel
}

\begin{abstract}
Shared-memory programmers traditionally assumed Sequential Consistency (SC), but modern systems have relaxed memory consistency. Here, the trend in languages is toward Data-Race-Free (DRF) models, where, assuming annotated synchronizations and the program being well-synchronized by those synchronizations, the hardware and compiler guarantee SC. However, legacy programs lack annotations, so even well-synchronized (legacy DRF) programs aren't recognized. For legacy DRF programs, we can significantly prune the set of memory orderings determined by automated fence placement by automatically identifying synchronization reads. We prove our rules for identifying them conservatively, implement them within LLVM, and observe a $30 \%$ average performance improvement over previous techniques.
\end{abstract}

Categories and Subject Descriptors: B.3 [Hardware]: Memory Systems; D.3.4 [Programming Languages]: Processors-Compilers

General Terms: Performance, Reliability

Additional Key Words and Phrases: Fence placement, relaxed memory models

ACM Reference Format:

Andrew J. McPherson, Vijay Nagarajan, Susmit Sarkar, and Marcelo Cintra. 2015. Fence placement for legacy data-race-free programs via synchronization read detection. ACM Trans. Archit. Code Optim. 12, 4, Article 46 (December 2015), 23 pages.

DOI: http://dx.doi.org/10.1145/2835179

\section{INTRODUCTION}

\subsection{The Problem}

A memory consistency model is at the heart of shared memory concurrency and specifies the value that each read in the program can return. Sequential Consistency (SC) [Lamport 1979], in which each read returns the last value written to that location in a global order found by interleaving the actions of each thread, is arguably the most intuitive of memory models [Ceze et al. 2007; Hill 1998; Lee and Padua 2001; Singh et al. 2012].

Unfortunately, as is now well-known, modern hardware does not provide SC to the programmer. Instead, different hardware architectures produce different varieties of

Extension of Conference Poster [McPherson et al. 2015]. This version additionally provides proof of our signatures, a worked example showing the impact of our technique, algorithms used in our implementation, and more detailed results.

Authors' addresses: A. J. McPherson (current address), IBM Research - Zurich, Switzerland; email: amc@zurich.ibm.com; V. Nagarajan, School of Informatics, University of Edinburgh, United Kingdom; email: vijay.nagarajan@ed.ac.uk; S. Sarkar, School of Computer Science, University of St. Andrews, United Kingdom; email: ss265@st-andrews.ac.uk; M. Cintra, Intel, Germany; email: marcelo.cintra@intel.com.

Permission to make digital or hard copies of part or all of this work for personal or classroom use is granted without fee provided that copies are not made or distributed for profit or commercial advantage and that copies show this notice on the first page or initial screen of a display along with the full citation. Copyrights for components of this work owned by others than ACM must be honored. Abstracting with credit is permitted. To copy otherwise, to republish, to post on servers, to redistribute to lists, or to use any component of this work in other works requires prior specific permission and/or a fee. Permissions may be requested from Publications Dept., ACM, Inc., 2 Penn Plaza, Suite 701, New York, NY 10121-0701 USA, fax +1 (212) 869-0481, or permissions@acm.org.

(c) 2015 ACM 1544-3566/2015/12-ART46 $\$ 15.00$

DOI: http://dx.doi.org/10.1145/2835179 
relaxed consistency behavior [Adve and Gharachorloo 1995]. Also, an agnostic compiler could perform optimizations that could violate SC.

The primary means by which the compiler can provide support is to insert appropriate fences to enforce sufficient orderings to restore SC. Each processor architecture provides different fences to enforce various types of orderings. The challenge is to insert sufficient fences to restore SC while at the same time not inserting too many. Fences are expensive because they limit many of the optimization opportunities available to hardware because of the relaxed memory consistency. Indeed, placing fences between every pair of accesses would guarantee SC but would be far too expensive.

The starting point of understanding the required placement of fences is the seminal Delay-set analysis of Shasha and Snir [1988]. They observed that, to ensure SC, it is not necessary to order all pairs of accesses. Only conflicting pairs of accesses (the delay sets) that can potentially lead to SC violations need to be ordered, where conflicting accesses are two accesses to the same address, at least one of which is a write. The memory orderings produced by Delay-set analysis are then subject to fence minimization [Lee and Padua 2001], which seeks to minimize the number of fences required to enforce these memory orderings.

One major issue that limits the practicality of Delay-set analysis is its reliance on alias analysis, which is notoriously imprecise for programs that make heavy use of pointers. In addition to this, scalability is also an issue for large programs. To overcome the scalability issue, approximations of Delay-set analysis using escape analysis have been developed, notably by the the Pensieve project [Fang et al. 2003; Sura et al. 2005]. More recently, attempts have also been made to address the scalability issue without resorting to escape analysis [Alglave et al. 2014], although recursion and dynamic thread creation continues to limit applicability. For either approach, however, the imprecision issue remains unresolved even with state-of-the-art alias analysis. This causes Delay-set analysis to produce a large number of superfluous orderings for real-world programs [Adve and Gharachorloo 1995; Lin et al. 2010; Singh et al. 2012].

\subsection{Our Approach}

We take a fresh look at fence placement. Our point of departure is that we do not seek to enforce SC for the general case. Instead, we insert sufficient fences to ensure that those memory accesses that are race free ${ }^{1}$ in the $\mathrm{SC}$ world continue to be race free in the relaxed world. To put it succinctly, we guarantee SC behavior only for race free accesses.

Our approach is based on the hypothesis that SC (which strongly orders all accesses) is not an end in itself to programmers; rather, it is enough for programmers to have SC semantics only for synchronization accesses (where synchronization accesses are those accesses that are used to guard other data accesses from racing). Therefore, it suffices if we identify such synchronization accesses and provide SC semantics for only those accesses. In order to understand this better, let us consider the two examples shown in Figure 1(a) and 1(b).

In the producer-consumer example shown in Figure 1(a), the programmer synchronizes using the flag variable to ensure that the read $b_{2}$ returns the value produced by $a_{1}$ (and not the old value). In this example, accesses $a_{2}$ and $b_{1}$ are synchronization accesses. Therefore, providing SC semantics to these accesses ensures that $b_{2}$ reads the correct value. The second example, shown in Figure 1(b), is a piece of code similar to that found in a relaxation solver [Chazan and Miranker 1969; Frommer and Szyld 2000] in which the four accesses involved are unsynchronized accesses (by design).

\footnotetext{
${ }^{1} \mathrm{~A}$ memory access is said to be race free if, in all legal SC executions, it is ordered with its conflicting accesses in each execution via the ordering chain introduced in Section 3 (following Gharachorloo [1995]).
} 


\begin{tabular}{|l|l|}
\hline \multicolumn{2}{|c|}{ (a) } \\
\hline P1 & P2 \\
$a_{1}:$ data $=1 ;$ & \\
$a_{2}:$ flag $=1 ;$ & $b_{1}:$ while $($ flag $==0) ;$ \\
& $b_{2}: x=$ data $;$ \\
\hline
\end{tabular}

\begin{tabular}{|l|l|}
\hline \multicolumn{2}{|c|}{$(\mathrm{b})$} \\
\hline $\mathrm{P} 1$ & $\mathrm{P} 2$ \\
& \\
$a_{1}: x=C_{1} ;$ & \\
$a_{2}: y=C_{2} ;$ & $b_{1}:$ local $_{2}=y$ \\
& $b_{2}:$ local $_{1}=x$ \\
\hline
\end{tabular}

Fig. 1. Examples of well-synchronized (a) and not well-synchronized (b) programs.

Here, it is permissible for the accesses in either thread to be reordered; for example, for the read of $x$ in P2 to return a stale value (occurring before $a_{1}$ in P1) while $b_{1}$ reads the value written by $a_{2}$. In other words, they are data races, albeit benign in this case. Therefore, providing SC semantics to such unsynchronized accesses is not required.

Although we do not promise SC in general, it is important to note that our approach guarantees SC for well-synchronized programs (i.e., legacy data-race-free programs). ${ }^{2}$ Figure 1(a) is an example of a well-synchronized program, whereas Figure 1(b) is not.

Our approach is similar in spirit to data-race-free (DRF) programming models, which form the basis of recent concurrent programming language models such as the C11 concurrency model [Batty et al. 2011; Boehm and Adve 2008] and the Java Memory Model specification [Manson et al. 2005]. This is a programming model that gives semantics to only DRF programs, programs in which all potentially racing operations (including synchronization operations and nonsynchronizing ones) are correctly labelled. In return for this discipline, the system (hardware + compiler) guarantees SC. However, legacy programs lack these labels, and our approach can be thought of as an automatic method for discovering such labels for legacy programs. Note, however, that we can only detect synchronization operations and not the other races (such as those benign races in Figure 1(b)).

\subsection{Our Solution}

We look for ways to conservatively identify synchronization operations. If we can be relatively precise, we can prune unnecessary orderings found by more traditional approaches. The existing fence minimization techniques can then be applied on the pruned orderings to achieve improved performance. An alternative application would be to use this identification to provide minimal annotations to make the program DRF, such that a compliant compiler and the hardware will prevent incorrect reorderings.

We have identified two signatures, at least one of which must be fulfilled for a read to be a synchronization (i.e., an acquire operation):

- Control acquire: a read feeds its value to a predicate tested for in a branch in its forward slice.

-Address acquire: a read feeds its value into the calculation of the address used by a data access in its forward slice.

We formally prove that at least one of these must hold for a read to be an acquire. The second signature (address acquire) is less prevalent and, in particular, is observed to appear along with the first signature (control acquire) in all cases in our experiments. We do not improve the identification of releases and, as in Pensieve, conservatively consider every shared write (escaping write) to be a release.

To evaluate the significance of our contribution, we next design and implement practical algorithms for identifying the acquires. Our simpler first algorithm (Control)

\footnotetext{
${ }^{2}$ More formally, these refer to a class of programs whose behavior is characterized by values returned by only those reads that are race free under SC.
} 
detects only control acquires and does not do interprocedural flow analysis (which is expensive). This does mean that the algorithm theoretically does not detect all acquiring reads. In particular, it does not detect cases where the acquiring read and the branch (both of which intuitively form the acquire) are split across two functions. ${ }^{3}$ We believe this will only rarely, if ever, be violated. In all our experiments, we never see such a split, though contrived programs can be written.

Control will also not detect address acquires. Again, in all our experiments, we have never seen an address acquire that is not also a control acquire. However, for completeness, we also develop a more conservative variant of our algorithm (Address+Control). This variant detects address acquires in addition to control acquires. As with the Control variant, we do not detect cases where the read and the use as an acquire is split across multiple functions.

We implemented our analysis in LLVM and applied it to the SPLASH-2 benchmark suite and a set of lock-free programs. Our experimental results show that, on average, Control reduces the number of orderings considered by $66 \%$ on average. Applying a fence minimization technique, this translates to an average of $62 \%$ fewer fences on x86TSO and up to $2.64 \times$ speedup over an existing practical technique. Address+Control on average reduces orderings considered by $32 \%$, fences placed by $27 \%$, and produces speedup of up to 1.54 .

The contributions of this article are:

(1) We improve fence insertion for legacy programs by discovering synchronization read operations.

(2) We prove that for all the necessary orderings (essential orderings) involving a synchronization read, the read has to satisfy at least one of two specific signatures: (i) that there is a conditional branch whose condition depends on the value returned by the read in the forward slice of the read and (ii) that a read provides the address for a subsequent access that would otherwise be unknown.

(3) We propose two practical algorithms: Control that detects only control acquires and Address+Control that detects both address and control acquires. Both algorithms work in the presence of pointers.

(4) We implement our algorithm within LLVM and observe an average of $62 \%$ fewer fences and up to $2.64 \times$ speedup over an existing practical technique with the simpler algorithm and an average of $27 \%$ fewer fences and up to $1.54 \times$ speedup with the conservative algorithm.

\section{OUR APPROACH}

\subsection{Fence Placement: Background}

The starting point of understanding the required placement of fences is Shasha and Snir's Delay-set analysis. Its key insight is that not all pairs of memory accesses need to be ordered to ensure SC. Only pairs of memory accesses that conflict with accesses from other threads, potentially leading to (minimal) SC violations known as critical cycles, need to be ordered. Identifying such critical cycles, however, presents a scalability issue on real-world programs (with pointers, recursion etc.) because it relies on heavyweight interprocedural static analysis. To overcome this, practical tools such as Pensieve [Fang et al. 2003; Sura et al. 2005] approximate Delay-set analysis.

This conservative approximation [Fang et al. 2003] is attained by such tools in a two-step process. First, a conservative thread-escape analysis is performed on each access in a function to determine a set of potentially escaping accesses, $E$. Second, for

\footnotetext{
${ }^{3}$ Note that the data accesses that the acquire protects are subject to no such assumption and can be located in a separate function.
} 
Table I. Sufficient Orderings for Correctness in a DRF Program

\begin{tabular}{|c|l|}
\hline$r / w \rightarrow w_{r e l}$ & $\begin{array}{l}\text { All reads and writes before the release (in program order) should be ordered } \\
\text { before the release }\end{array}$ \\
\hline$r_{a c q} \rightarrow r / w$ & $\begin{array}{l}\text { All reads and writes after the acquire (in program order) should be ordered } \\
\text { after the acquire }\end{array}$ \\
\hline$w_{r e l} / r_{a c q} \rightarrow w_{r e l} / r_{a c q}$ & All synchronization operations should be ordered among themselves \\
\hline
\end{tabular}

$u, v \in E$, if analysis of the control flow graph shows that $v$ can occur after $u$, then an ordering, $u \rightarrow v$, is recorded.

Although this does generate a correct set of orderings, it produces a large number of false positives due to the thread-escape analysis being necessarily conservative. In practice, this means that all references to memory that cannot be proved to be restricted to the local function must be marked as potentially escaping.

Once a set of orderings has been identified, these orderings are fed as input to a fence minimization algorithm. Such an algorithm will determine where to minimally place fences to ensure that all the orderings are enforced. It may also distinguish between types of orderings to minimize the cost of enforcement. This can be achieved by using different types of fences or compiler directives, depending on the memory consistency model of the target architecture. For example, x86-TSO only requires orderings of the type $w \rightarrow r$ to be enforced with full memory fences because other orderings are enforced by the hardware. These other orderings, however, still have to be preserved during the compilation (optimization) process.

\subsection{Fence Placement for DRF Programs}

Now let us consider fence placement for a DRF program. Recall that in a DRF program, synchronization is achieved using special memory operations-a write known as a release and a read known as an acquire-such that there are no races among data operations. This implies that, given such a well-synchronized program without data races, enforcing the orderings defined in Table I is sufficient to ensure correctness [Adve 1993].

In more detail, the first rule requires that all accesses to shared data must be performed before a release. Similarly, the second rule requires that all accesses to shared data must be performed only after an acquire. These two, combined with the third rule, ordering all acquires and releases, ensures correctness.

With precise information as to which of the reads (writes) are acquires (releases), determining the minimal set of required orderings is trivial. Specifically, orderings that do not conform to one of the definitions in Table I could be safely ignored. The set of required orderings could then be fed as input to a fence minimization algorithm.

\subsection{Identifying Acquires for Legacy DRF}

There exists, however, a large body of (legacy) code that is correctly synchronized, but the distinction between a read $(r)$ and an acquiring read $\left(r_{a c q}\right)$ and a write $(w)$ and a release $\left(w_{\text {rel }}\right)$ is not made explicit by the programmer. We call such programs Legacy DRF.

One way to perform fence placement for such programs is to treat it like a general multithreaded program; that is, use Delay-set analysis (or its conservative approximation) followed by fence minimization techniques. Our key insight is that we can do better if we can conservatively identify synchronization operations. In this article, we focus on detecting acquires.

\footnotetext{
${ }^{4}$ Weaker models that relax some of these requirements, such as $\mathrm{RC}_{P C}$ [Adve and Gharachorloo 1995] in hardware and C11 [Batty et al. 2011; Boehm and Adve 2008] at the language level, also exist.
} 


\begin{tabular}{|c|c|c|c|c|c|}
\hline \multicolumn{2}{|c|}{ Legacy DRF Code } & \multicolumn{2}{|c|}{ Delay-set Fence Placement } & \multicolumn{2}{|c|}{ Pruned Orderings Fence Placement } \\
\hline $\begin{array}{l}\mathrm{P} 1 \\
a_{1}: x=\end{array}$ & $\begin{array}{l}\mathrm{P} 2 \\
b_{1}: * p 1=\end{array}$ & $\begin{array}{l}\mathrm{P} 1 \\
a_{1}: x=\end{array}$ & $\begin{array}{l}\mathrm{P} 2 \\
b_{1}: * p 1=\end{array}$ & $\begin{array}{l}\mathrm{P} 1 \\
a_{1}: x=\end{array}$ & $\begin{array}{l}\mathrm{P} 2 \\
b_{1}: * p 1=\end{array}$ \\
\hline$a_{2}:=y$ & $b_{2}:=* p 2$ & $\overline{a_{2}}:=y$ & $\overline{b_{2}}:=* p 2$ & $a_{2}:=y$ & $b_{2}:=* p 2$ \\
\hline & & $-(F 2)-$ & & $-(F 2)-$ & \\
\hline$a_{3}:$ flag $=1$ & $b_{3}:$ while $($ flag $!=1)$ & $a_{3}:$ flag $=1$ & $\begin{array}{l}b_{3}: \text { while }(\text { flag } !=1) \\
-(F 4)-\end{array}$ & $a_{3}:$ flag $=1$ & $\begin{array}{l}b_{3}: \text { while }(\text { flag! }=1) \\
-(F 4)-\end{array}$ \\
\hline & $b_{4}: y=$ & & $\begin{array}{l}b_{4}: y= \\
-(F 5)-\end{array}$ & & $b_{4}: y=$ \\
\hline & $b_{5}:=x$ & & $b_{5}:=x$ & & $b_{5}:=x$ \\
\hline
\end{tabular}

Fig. 2. An example of (full) fence placement on legacy DRF code for delay-set and pruned orderings.

We prove that for a read to be an acquire it must match at least one of two signatures. The first is that there exists a branch whose predicate is data-dependent on the read, in the forward slice of that read. The second is that the read contributes its value to an address calculation for a data access in its forward slice. Any read that fails to satisfy at least one of these signatures cannot be an acquire.

Intuitively, an acquire is a read that determines if shared data can be accessed. This necessarily involves either checking the value read and acting on it (the first signature) or providing the address of data, which would otherwise be inaccessible (the second signature). A formal proof of these assertions can be found in Section 3.

By applying the two signatures to every read that may be thread-escaping, we determine a subset that includes every potential acquire.

Having identified a conservative subset of the shared reads as potential acquires, we are able prune the orderings. Starting from the set of orderings given by Delay-set analysis (or its approximation that uses escape analysis), we prune all those orderings that do not adhere to one of the definitions in Table I. Despite not identifying a subset of the shared writes and therefore having to consider all shared writes as releases, we are still able to prune a number of potentially expensive orderings.

Specifically, any ordering of the form $r_{1} \rightarrow r_{2}$ requires at least $r_{1}$ to be an acquire to avoid being pruned; that is, it must be of the form $r_{a c q} \rightarrow r$. Similarly, any ordering of the form $w_{1} \rightarrow r_{2}$ requires $r_{2}$ to be an acquire to avoid being pruned (i.e., of the form $\left.w \rightarrow r_{a c q}\right)$.

This reduced number of orderings is provided as (an improved) input to a fence minimization algorithm, resulting in a much reduced number of fences.

\subsection{An Example}

To illustrate the impact of pruning orderings, we now demonstrate the application of Delay-set analysis to a section of legacy DRF code and the fences that this would require. Then, using the acquire signatures and applying the pruning rules just defined, we determine the reduced set of fences required to enforce the remaining orderings.

In Figure 2, we present a section of legacy DRF code that contains a busy-waiting synchronization. For the purposes of this example, we assume that alias analysis has determined that $* p 1$ and $* p 2$ may potentially alias with both $x$ and $y$, but not flag. If one were to apply Delay-set analysis, the following orderings would be determined to avoid the following critical cycles:

$-a_{1} \rightarrow a_{3}, b_{3} \rightarrow b_{5}:$ to avoid $\left(a_{1}, a_{3}, b_{3}, b_{5}, a_{1}\right)$.

$-a_{2} \rightarrow a_{3}, b_{3} \rightarrow b_{4}$ : to avoid $\left(a_{2}, a_{3}, b_{3}, b_{4}, a_{2}\right)$.

$-a_{1} \rightarrow a_{2}, b_{4} \rightarrow b_{5}:$ to avoid $\left(a_{1}, a_{2}, b_{4}, b_{5}, a_{1}\right)$.

$-a_{1} \rightarrow a_{2}, b_{1} \rightarrow b_{2}$ : to avoid $\left(a_{1}, a_{2}, b_{1}, b_{2}, a_{1}\right)$.

In the final cycle, our assumption regarding $* p 1$ and $* p 2$ potentially aliasing with $x$ and $y$ but not flag comes into play. 


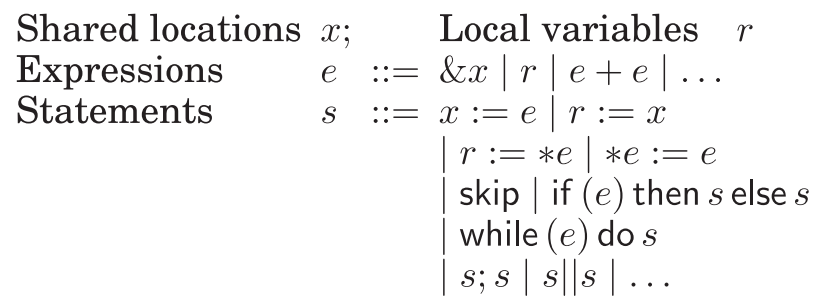

Fig. 3. The programming language for proofs.

Using these orderings as input to a fence minimization algorithm, five (full) fences are required to be placed to enforce the orderings. Placement of these fences is shown as "Delay-set Fence Placement" in Figure 2.

Pruning the orderings by applying the signatures defined in Section 2.3, we find that only the following remain:

$-a_{1} \rightarrow a_{3}, b_{3} \rightarrow b_{5}:$ to avoid $\left(a_{1}, a_{3}, b_{3}, b_{5}, a_{1}\right)$.

$-a_{2} \rightarrow a_{3}, b_{3} \rightarrow b_{4}$ : to avoid $\left(a_{2}, a_{3}, b_{3}, b_{4}, a_{2}\right)$.

Of the orderings that have been pruned: $a_{1} \rightarrow a_{2}, b_{1} \rightarrow b_{2}$ and $b_{4} \rightarrow b_{5}$ are not required as neither $a_{2}, b_{2}$, nor $b_{5}$ are acquires. Using this reduced set of orderings as input to the same fence minimization algorithm, only two (full) fences are required to be placed. These fences are shown as "Pruned Orderings Fence Placement" in Figure 2.

$F 1, F 3$, and $F 5$ are no longer required and have been removed. However, $F 2$ and $F 4$ are still required. Together they prevent $\left(a_{1}, a_{3}, b_{3}, b_{5}, a_{1}\right)$ and $\left(a_{2}, a_{3}, b_{3}, b_{4}, a_{2}\right)$, with $F 2$ enforcing $a_{1} \rightarrow a_{3}$ and $a_{2} \rightarrow a_{3}$, and $F 4$ enforcing $b_{3} \rightarrow b_{4}$ and $b_{3} \rightarrow b_{5}$.

In summary, we expect our signatures to considerably reduce the number of orderings that need to be enforced. With reference to our example, there are three major benefits:

-Acquire detection allows us to avoid enforcing many orderings that are not necessary (e.g., data $\rightarrow$ data orderings such as $a_{1} \rightarrow a_{2}$ and $b_{4} \rightarrow b_{5}$ ) since the program is wellsynchronized.

- The inherent imprecision of Delay-set analysis (or its approximation) in the presence of pointers results in the enforcement of orderings that are not necessary. Acquire detection allows us to prune some of these orderings (e.g., $b_{1} \rightarrow b_{2}$ ).

- This reduction in the number of orderings allows a fence minimization algorithm to place fewer fences (in this case, not placing $F 1, F 3$ and $F 5$ ).

\section{CORRECTNESS OF ACQUIRE SIGNATURES}

In this section, we formally prove the basis of our preceding assertions; that is, a synchronization read (acquire) matches (at least) one of two signatures. One is that in its forward slice, there must be a conditional dependent on the value returned by the read. The other is that the acquire reads a value used in determining the address of a subsequent access in the forward slice of the acquire.

Language. For concreteness, we define our programming language to be a simple multithreaded "while" language with pointers. Expressions $e$ are pure, defined as making no shared-memory loads or stores, although local variables (marked with an $r$ ) are allowed. Statements then can dereference pointers and load from and store to shared-memory locations, either explicitly or via pointers. The language is presented in Figure 3.

This tiny language captures all the essential features needed for our results. Note that in comparison to a full-scale language such as C, key simplifications are that all 
shared-memory loads and stores from a single thread are explicitly sequenced and that function calls and returns are ignored. These calls can, however, be handled via inlining, and their exclusion here does not affect the statements proven. We do exclude self-modifying code because, absent a Just-In-Time compiler, we do not believe it can be reliably handled. Additionally, we are unaware of any fence placement system that claims to support it. We also ignore read-modify-writes, but these can easily be added to the proof presented later by considering them to be a read followed by a write to the same location.

Intended Behavior. Given a program in the given language, we assume that there is some intended marking of accesses (shared-memory loads and stores) into data and synchronization accesses. Data accesses are programmer-intended accesses; more formally, the behavior intended by the programmer is defined by the values read by the data reads. The rest of the accesses are assumed to be synchronization accesses; these are assumed to be written only to make sure there are no races on the data accesses. Following standard practice, we call synchronization reads acquire reads and synchronization writes release writes.

Behavior under SC. A SC execution is an execution trace (a linear order of read and write actions) that is a free interleaving of thread-wise actions, such that actions belonging to any thread appear in the execution trace in the order they occur in that thread, and each memory read reads the value of the last write to that location in the trace. Note that, in general, a single access in the program might lead to one or more actions in the trace (due to loops) or none (in case of a conditional). There is a straightforward way of associating each action in the trace to at most one program access, and we associate the corresponding kind (data or synchronization) of program access to the actions. Of course, because there might be several possible interleavings, a program has a set of allowed SC executions. For each such execution, we intuitively consider the results of the execution to be the values returned by the data reads. We formally consider the intended behavior of the program to be the set of data read actions of any possible SC execution.

Behavior under relaxed consistency. A program actually executes not on an SC machine but on a machine with relaxed consistency. We follow the approach of Adve and Hill [1990] (the approach of Gharachorloo [1990] is very similar) and define that a program is correct if and only if it has no more behavior in a relaxed consistency setting than in the SC world.

We define happens-before following Gharachorloo [1995] by first defining conflict order and program order. Define conflict order $\stackrel{c o n}{\longrightarrow}$ to be an order relation between conflicting actions in an execution (the order says one happens before the other), where two actions conflict if they are to the same address and at least one is a write. In particular, a write is conflict-ordered before a read if the read reads from that write. Also, there is an obvious program order relation $\stackrel{p o}{\rightarrow}$ between actions from the same thread.

Given two actions $u$ and $v, u$ happens-before $v$ (written $u \stackrel{h b}{\rightarrow} v$ ) in that execution if either $u \stackrel{p o}{\longrightarrow} v$ or $u \stackrel{p o}{\longrightarrow} w_{1} \stackrel{\text { con }}{\longrightarrow} r_{1} \stackrel{p o}{\longrightarrow} w_{2} \stackrel{c o n}{\longrightarrow} r_{2} \ldots w_{n} \stackrel{c o n}{\longrightarrow} r_{n} \stackrel{p o}{\longrightarrow} v$. We consider only executions in which each synchronization read reads from the last write to that location in happens-before. The behavior of a program is determined by the data reads (value and location) of all such executions.

Well-synchronized programs. We call a program (legacy) DRF if, in all executions (where synchronization reads read from the last write in happens-before as above), all conflicting data actions are ordered by $\stackrel{h b}{\rightarrow}$. It has been proved [Adve and Hill 1990; 


\begin{tabular}{|ll|}
\hline \multicolumn{1}{|c|}{ MP } \\
\hline P1 & P2 \\
$a_{1}: x:=$ & \\
$a_{3}:$ flag $:=1$ & $b_{3}:$ while $\left(r_{1} !=1\right)\left\{r_{1}:=\right.$ flag $\}$ \\
& $b_{5}: r=x$ \\
\hline
\end{tabular}

Fig. 4. The MP example.

Gharachorloo et al. 1990] that DRF programs have no more behavior in this sense than SC behavior of the same program. However, since legacy programs do not have explicit markings of data and synchronization, and to avoid confusion with the standard DRF notion, we equivalently call legacy DRF programs well-synchronized.

Ordering edges: Essential and nonessential. We call a program order edge essential if ignoring that edge allows a data read to read a value not possible under SC and all other program order edges nonessential. Thus, enforcing all essential program order edges is sufficient to preserve SC behavior for the data reads.

We now prove a happens-before characterization of essential edges. Specifically, we prove that an edge in a well-synchronized program (i.e., a (legacy) DRF program) is essential if and only if ignoring that edge in happens-before as just defined allows an execution with a data race.

LEMma 3.1. For a program that is DRF for a certain mapping, and $U \rightarrow V$ a program order edge, the edge is essential if and only if deleting $U \rightarrow V$ from happens-before allows an execution with a data race involving a read and write.

Proof. Both directions follow easily from unfolding the definitions.

For one direction, ignoring an essential edge allows a data read to read a value not possible under SC. That data read and the write it reads from must be in a data race since, if they are ordered via happens-before, then the read is still possible under SC.

In the other direction, suppose deleting $U \rightarrow V$ from happens-before allows an execution with a data race between a read and a write. Consider that read. Since the program is well-synchronized (i.e., no data races before removing that edge), the read could not have read from that write.

Intuitively, if we disregard an essential ordering edge, the program is no longer DRF, and thus the DRF guarantees of Adve and Hill [1990] and Gharachorloo et al. [1990] do not apply. In that case (disregarding essential orderings), there will be data reads observable that are not possible in SC executions. This happens-before characterization is easier to prove because we can now analyze the shapes of happens-before.

Informal explanation. We are now in a position to give the formal proof of our main result, Theorem 3.1. Before that, to orient the reader, we give the main idea of the proof informally.

The key insight is that if there is an essential ordering involving an acquire, then the acquire must have been guarding a data access; only then will relaxing the given ordering result in a data race (and thus, by Lemma 3.1, non-SC behavior for the data reads). We illustrate three different ways in which an acquire can guard data. The formal proof will essentially say that these are the only cases to consider, which allows us to safely deduce the acquire signatures.

The first way in which an acquire can guard data is illustrated via the classic Producer-Consumer or MP (Figure 4). Here, the data access (of $x$ ) is guarded by controldependency; that is, control only flows to it if the (acquire) read of flag reads 1. 


\begin{tabular}{|l|}
\hline \multicolumn{2}{|c|}{ MP with Pointers } \\
\hline Initially $z=0, y=\& z, x=0$ \\
$\begin{array}{ll}\mathrm{P} 1 & \mathrm{P} 2 \\
a_{1}: x= & \\
a_{3}: y=\& x & b_{3}: r=y \\
& b_{5}: r_{1}=* r\end{array}$ \\
\hline
\end{tabular}

Fig. 5. The MP example with pointer arithmetic.

\begin{tabular}{|l|l|}
\hline \multicolumn{3}{|c|}{ Dekker } \\
\hline P1 2 \\
$a_{1}: x:=1$ & $b_{1}: y:=1$ \\
$a_{2}:$ if $(y==0)\{$ & $b_{2}:$ if $(x==0)\{$ \\
$a_{3}: \quad$ touch $\left.z\right\}$ & $b_{3}:$ touch $\left.z\right\}$ \\
\hline
\end{tabular}

Fig. 6. The Dekker example.

The second way is when the value read by the acquire is used to calculate the address touched by the data access (i.e., it only reads from the location if the acquire read a certain value). This could happen in the example in Figure 5, an example adapted from Gharachorloo. Here, $y$ (analogous to flag above) stores the address of $z$ initially, and the second read on the second thread reads from $x$ only if the prior read reads $x$ (otherwise it reads from $z$ ).

The third possible way is to have some form of mutual exclusion in which the data access is in a critical region. In this case (seen in the Dekker's example in Figure 6), the data access is prevented from performing in an execution where the synchronization read reads the wrong value.

Formal proofs. Given a program, and if we knew the marking into data and synchronization, we call two accesses potentially racing if they are on different threads, at least one of them is a data write, and they are either statically to the same location or at least one of them is to a statically unknown location (this can happen if it is to a location derived from a value read before on the same thread).

Lemma 3.2. For two potentially racing accesses $U$ and $V$ in the program and any legal execution Xaccording to the relaxed consistency model, at least one of the following must happen:

(1) $U$ and $V$ correspond to two actions that form a data race in $X$;

(2) $U$ and $V$ correspond to actions $u$ and $v$, respectively, in $X$ that are ordered $u \stackrel{p o}{\rightarrow}$ $w_{1} \stackrel{\text { con }}{\longrightarrow} r_{1} \stackrel{p o}{\longrightarrow} w_{2} \stackrel{\text { con }}{\longrightarrow} r_{2} \ldots w_{n} \stackrel{\text { con }}{\longrightarrow} r_{n} \stackrel{p o}{\longrightarrow} v$ in $X$

(3) $U$ and $V$ correspond to actions $u$ and $v$, respectively, in $X$ that are to different locations (this can only happen for statically unknown locations);

(4) at least one of $U$ and $V$ do not correspond to any actions in $X$;

Proof. Immediate from the definitions of data races and happens-before.

Lemma 3.2 intuitively says that for static program accesses that potentially race, in any execution either there is an actual race or there is a proper happens-before ordering (such as in Figure 4) between the actions corresponding to the race, or one or 
the other access is to a different locations (such as in Figure 5) or absent altogether (such as in Figure 6).

LEMMA 3.3. For all essential orderings that are of the following form:

(1) $R \rightarrow A$, where $R$ is an acquire and $A$ is a subsequent access; or

(2) $W \rightarrow R$, where $W$ is a write and $R$ is a subsequent acquire,

the value read from the acquire must feed into:

- Either a conditional that guards a subsequent access;

-Or an address computation that determines the location of a subsequent access.

Proof. Given the essential ordering edge in the premise of the theorem, it can be of two types: $\mathrm{R} \rightarrow \mathrm{A}$, or $\mathrm{W} \rightarrow \mathrm{R}$. Consider disregarding this ordering edge in happensbefore. Since the ordering edge is essential, by Lemma 3.1, there is a data race in some execution. Call that execution $X$, and consider the two data accesses $U$ and $V$ involved in the race. Since they correspond to racing actions in an execution, they must be potentially racing accesses. Consider the execution $Y$ with the ordering edge present; otherwise, it is the same as $X$, except that because reads may read different values, some actions may not occur or occur with different values in $Y$ than in $X$. Apply Lemma 3.2 to the legal execution $Y$. Then one of the four cases must apply.

Case 1. In $Y, U$ and $V$ correspond to two actions $u$ and $v$ that form a data race. Since the program is assumed DRF, and $Y$ is a legal execution, this case cannot occur.

Case 2. In $Y, U$ and $V$ correspond to actions $u$ and $v$, respectively, in $X$ that are ordered $u \stackrel{p o}{\longrightarrow} w_{1} \stackrel{c o n}{\longrightarrow} r_{1} \stackrel{p o}{\longrightarrow} w_{2} \stackrel{\text { con }}{\longrightarrow} r_{2} \ldots w_{n} \stackrel{\text { con }}{\longrightarrow} r_{n} \stackrel{p o}{\longrightarrow} v$ in $X$. The ordering edge in question must occur in this chain. Since there is no W $\rightarrow R$ ordering edge in this chain, the essential ordering edge we are dealing with must be of the form $R \rightarrow A$. We now see where the action corresponding to $\mathrm{R}$ occurs in this chain. It cannot be the first step $\left(u \stackrel{p o}{\longrightarrow} w_{1}\right)$ since $u$ is a data access. It can be $r_{n}$ in the last step $\left(r_{n} \stackrel{p o}{\longrightarrow} v\right)$ or $r_{i}$ in an intermediate thread $\left(r_{i} \stackrel{p o}{\rightarrow} w_{i+1}\right)$. In each case, $\mathrm{R}$ reads the value of a synchronization write in this execution $Y$. Furthermore, $v$ or $w_{i+1}$, respectively, is the access $\mathrm{A}$ in question. Consider now a different execution where $R$ does not read the value of the same synchronization write. Then it must be the case that either A does not occur or A exists but accesses a different location, since otherwise the ordering chain does not exist and the program has a race. Thus, either $\mathrm{R}$ feeds into a conditional guarding $\mathrm{A}$ or is used to calculate the address touched by $\mathrm{A}$, as required.

Case 3. $U$ and $V$ correspond to actions $u$ and $v$, respectively, in $Y$ that are to different locations.

Since $U$ and $V$ correspond to racing actions $u^{\prime}$ and $v^{\prime}$ in $X$, at least one of the pairs $\left(u, u^{\prime}\right)$ and $\left(v, v^{\prime}\right)$ must be to different locations. Without loss of generality, let $u$ and $u^{\prime}$ be to different locations. Then, $U$ must be to a statically unknown location that is in fact different in $X$ and $Y$. Since $X$ differs from $Y$ in that the essential ordering edge (either $\mathrm{R} \rightarrow \mathrm{A}$ or $\mathrm{W} \rightarrow \mathrm{R}$ ) is not required, in either case, the calculation of the location for $U$ must be derived from the value returned by $\mathrm{R}$.

Case 4. At least one of $U$ and $V$ do not correspond to any actions in $Y$.

Without loss of generality, let there be no actions corresponding to $U$ in $Y$. Since $U$ corresponds to an action $u$ in $X, U$ must be guarded by a conditional that is true in $X$ but not in $Y$. Since $X$ differs from $Y$ in that the essential ordering edge (either $\mathrm{R} \rightarrow$ A or $\mathrm{W} \rightarrow \mathrm{R}$ ) is not required, in either case, this conditional must be derived from the value returned by $R$.

THEOREM 3.1. For all essential orderings involving an acquire $R$, the value read from the acquire must feed into: 
Table II. Breakdown of the Types of Acquires Found in Common Synchronization Kernels; Notably, No Acquires Are Found to Only Meet the Address Signature

\begin{tabular}{|l|c|c|c|l|}
\cline { 2 - 4 } \multicolumn{1}{c|}{} & \multicolumn{3}{c|}{ Acquires } & \multicolumn{1}{c|}{ Source } \\
\cline { 2 - 4 } \multicolumn{1}{c|}{} & Addr & Ctrl & Pure Addr & \\
\hline Chase Lev WSQ & $\checkmark$ & $\checkmark$ & $\mathbf{X}$ & [Chase and Lev 2005] \\
\hline Cilk-5 WSQ & $\mathbf{X}$ & $\checkmark$ & $\mathbf{X}$ & [Frigo et al. 1998] \\
\hline CLH Lock & $\checkmark$ & $\checkmark$ & $\mathbf{X}$ & [Craig 1994] \\
\hline Dekker & $\mathbf{X}$ & $\checkmark$ & $\mathbf{X}$ & [Dijkstra 1965] \\
\hline Lamport & $\mathbf{X}$ & $\checkmark$ & $\mathbf{X}$ & [Lamport 1987] \\
\hline MCS Lock & $\checkmark$ & $\checkmark$ & $\mathbf{X}$ & [Mellor-Crummey and Scott 1991] \\
\hline Michael Scott LFQ & $\checkmark$ & $\checkmark$ & $\mathbf{X}$ & [Michael and Scott 1996] \\
\hline Peterson & $\mathbf{X}$ & $\checkmark$ & $\mathbf{X}$ & [Peterson 1981] \\
\hline Szymanski & $\mathbf{X}$ & $\checkmark$ & $\mathbf{X}$ & [Szymanski 1988] \\
\hline
\end{tabular}

-Either a conditional that guards a subsequent access;

-Or an address computation that determines the location of a subsequent access

Proof. The possible orderings involving an acquire $R$ are:

Case 1. $R_{1} \rightarrow R$, where $R_{1}$ should also be an acquire (since data $\rightarrow$ acquire ordering is not essential). Proof is from Lemma 3.3 (treating $R_{1}$ as the acquire, first form applies).

Case 2. $W \rightarrow R$, where $W$ is a write. Proof is from Lemma 3.3, second form applies.

Case 3. $R \rightarrow A$, where $A$ is any access. Proof is from Lemma 3.3, first form applies.

\section{IMPLEMENTATION}

In this section, we present two algorithms for identifying synchronization reads as used in our implementation. The first algorithm (Control) only identifies acquires that meet our control signature, whereas the second (Address+Control) is conservative because it additionally identifies acquires that only match our address signature.

Whereas conservatism demands application of the address signature, in practice, we find that only the control signature is required. In all the experiments we perform (see Section 5), we find no acquires that only meet the address signature. To reinforce this point, we performed an empirical study of nine common synchronization primitives, the results of which are presented as Table II. It is worth noting that these primitives represent common patterns used in synchronization; indeed, some underpin programs we examine later in Section 5. As we can see, acquires that match the control signature are far more prevalent. Although there are acquires that meet the address signature, all of those also meet the control signature.

We make one simplifying assumption in our implementations: That the synchronizing reads occur in the same function as the condition to which they lead. Whereas an interprocedural algorithm would be a necessary step to achieving soundness, such a guarantee would also require access to all libraries/functions used at compile time. We believe that this assumption is reasonable since it is extremely rare for these two operations, which intuitively form the synchronization, to be split across two functions (although it is possible to construct a contrived example). Indeed, in none of the implementations of the primitives examined (implementations for CLH Lock and MCS Lock from David et al. [2013], all others from Alglave et al. [2014]), nor the real programs examined in Section 5 is this separation found.

Both of the algorithms depend on an intraprocedural static slicer that performs the actual identification of the synchronizing reads; this is presented in Section 4.1. All the algorithms operate on infinite register load-store intermediate representations. We now examine each algorithm in detail before finally outlining the generation of orderings and the fence minimization algorithm to which we input them. We assume that the 


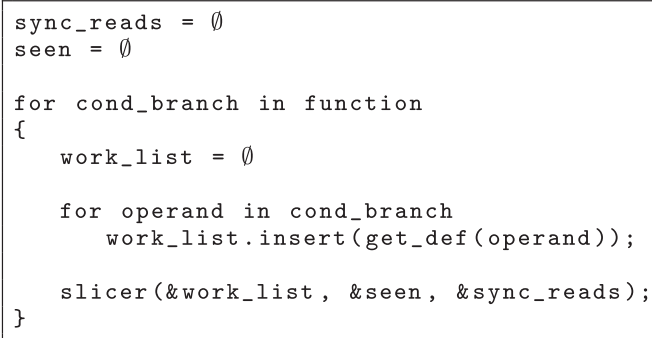

Listing 1. Algorithm Control, for matching the control signature.

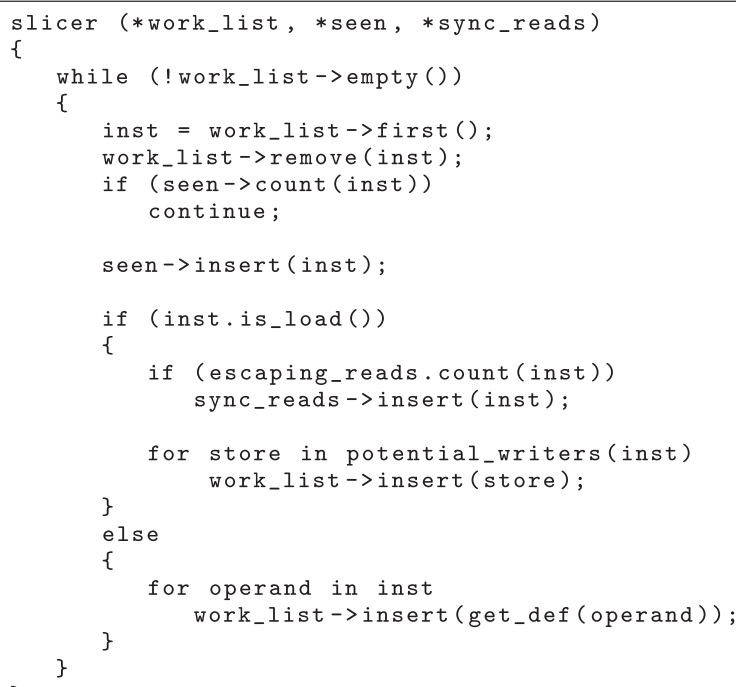

Listing 2. Algorithm for backward slicing and the registration of escaping reads contained in the slice.

set of escaping loads and stores has previously been identified using a thread-escape analysis as in Pensieve.

\subsection{Identifying Control Acquires}

The algorithm for identifying escaping reads that match our control signature (Control) is presented as Listing 1. To determine reads that meet our control signature, we must determine which reads have branches (conditions) in their forward slice. To determine this efficiently, the algorithm in fact focuses on each conditional branch and examines the reads in its backwards slice. For each conditional branch in a function, we retrieve the instructions that define the branch operands (Lines 8 and 9 ). Then we initiate the backwards slicer to populate sync_reads with escaping loads from the backwards slice of the conditional branch (Line 11).

Backward Slicing. The algorithm for backward slicing and populating sync_reads is presented as Listing 2. This algorithm performs a conservative intraprocedural backward slice from the initial contents of work_list. Every load found while processing the work_list is compared against the results of the prior escape analysis (Line 14), and, if escaping, added to sync_reads (Line 15). 


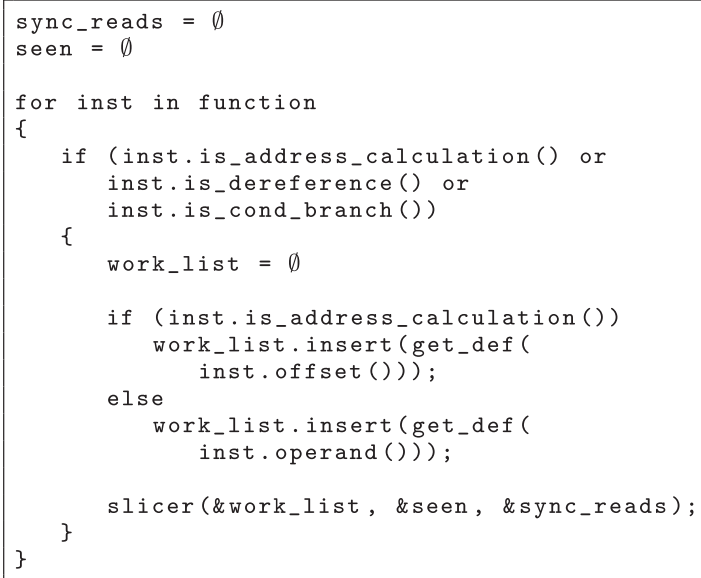

Listing 3. Algorithm Address+Control that identifies escaping reads that match either signature.

To ensure conservatism, whenever a load is found, alias analysis is used to find all stores in the function that potentially wrote the value being read (Line 17). These stores are added to the work_list to be processed later. For instructions that are not a load, each operand is processed, and the defining instructions of those operands are added to the work_list (Lines 22 and 23).

To avoid becoming trapped in cycles and to improve efficiency, both of the signature matching algorithms maintain sets of previously examined instructions, seen. The slicing algorithm is responsible for populating (Line 10) and checking against (Line 7) these sets. Once the work_list has been exhausted, the algorithm terminates.

\subsection{Identifying Both Control and Address Acquires}

As we previously stated, the algorithm presented in the previous sections provides sufficient coverage for all the real programs we have seen. It is, however, possible that an acquire only meets the address signature. To contend with this eventuality, we develop a more conservative variant of our algorithm (Address+Control), presented as Listing 3. This variant identifies escaping reads that meet either or both of the signatures identified.

As with the algorithm for the control signature, we use a backward slice. In addition to conditional branches, the slicing is performed from every instruction that is either a dereference or an address calculation. This ensures that any escaping reads that contribute to a value used as an address are added to sync_reads. In the case of a dereference, the slicer is applied to the operand of the instruction (i.e., the address; Line 16). In the case of an address calculation (e.g., a GetElementPtr instruction in LLVM IR), the offset is sliced (Line 13). As expected, these two cases often overlap with an address calculation in the backward slice and therefore subordinate to a dereference. Here again, the use of the seen set prevents reiteration.

\subsection{Generating Pruned Orderings}

Whichever algorithm has been used to populate sync_reads, the next step is the generation of orderings. Ordering generation is done in line with Pensieve, generating an ordering for every pair of variables in the set of potentially escaping loads and stores if there exists a path between them. Within a basic block, the order of statements gives a directed linear sequence of accesses. Whether there exists a path between basic blocks 
is determined prior to this process with an examination of the CFG to create a lookup table of reachability. This can then be queried during ordering generation.

The addition that we make to ordering generation is to prune $w \rightarrow r$ and $r \rightarrow r$ orderings that do conform to $w \rightarrow r_{a c q}$ and $r_{a c q} \rightarrow r$, respectively. The pruning is achieved by querying orderings of the form $w \rightarrow r$ and $r \rightarrow r$ for previously identified synchronizing reads.

\subsection{Fence Minimization}

Given the set of orderings to enforce, a fence minimization algorithm is used to place as few fences as possible while still enforcing all required orderings. To place fences, we use the locally optimized fence placement algorithm described in Fang et al. [2003]. The only alteration we make to this algorithm is to not automatically place a fence at the beginning of each function; such a fence is only placed if the function contains synchronizing reads. The rationale for placing this fence is to enforce interprocedural orderings: Under x86-TSO if the function contains no synchronizing reads then no interprocedural $w \rightarrow r$ orderings can terminate within the function, and the absence of a full fence does not affect correctness.

When determining full fence placement we need only consider orderings that the hardware will not enforce. Our technique is generally applicable, but, in our experiments, we target x86-TSO and therefore we only consider orderings of the form $w \rightarrow r$ because the other orderings are enforced automatically by hardware. However, to prevent incorrect reorderings by the compiler, we place compiler directives to enforce orderings of any other form. Specifically, these directives take the form of empty memoryclobbering assembly instructions that have no presence in the final binary but prevent reordering of memory-related statements around them. The same minimization algorithm is used here, with the decision as to whether to place a full fence or a compiler directive determined by whether the set of orderings that would be enforced contains one of the form $w \rightarrow r$.

\section{RESULTS}

We implemented our algorithms and a locally optimized fence minimization algorithm based on Fang et al. [2003] in LLVM 3.4.1. The programs were all compiled using the O2 optimizations.

Using a set of lock-free programs and the SPLASH-2 [Woo et al. 1995] benchmarks, we compare both the Control (control acquires only) and Address+Control (control and address acquires) variants of our approach with an implementation of Pensieve ${ }^{5}$ using locally optimized fence minimization (as described in Fang et al. [2003]). To establish a performance baseline, we also compare against a (minimal) manual fence placement. The lock-free programs are introduced in Table III.

It is worth noting that the programs considered are well-synchronized because they employ user-defined synchronization ${ }^{6}$ and hence require fences on relaxed models for correctness.

Our results are organized as follows. First, we examine how many reads marked as potentially thread-escaping our algorithms mark as an acquire, giving us a measure of the effectiveness of our technique. Second, we compare and break down by type the number of orderings generated by the naive and both variants of our approach. Third,

\footnotetext{
${ }^{5}$ We use the term Pensieve throughout this section to refer to the version presented in Fang et al. [2003] with locally optimized fence minimization, rather than the later Sura et al. [2005].

${ }^{6}$ Although the lock-free programs use user-defined synchronization exclusively, the SPLASH-2 programs make use of user-defined synchronization (in programs such as FMM [Tian et al. 2008] and Volrend [Nistor et al. 2010]) and also employ library calls to locks and barriers.
} 
Table III. Descriptions of the Lock-Free Programs Used

\begin{tabular}{|l|l|}
\hline Canneal & $\begin{array}{l}\text { A kernel that seeks to minimize routing cost for chip design using } \\
\text { cache-aware simulated annealing. This program was drawn from the } \\
\text { PARSEC suite [Bienia et al. 2008] and was run with the Simlarge input set. }\end{array}$ \\
\hline Matrix & $\begin{array}{l}\text { A parallel implementation of matrix multiplication that takes in two matrices } \\
\text { and outputs both potential matrix products. To allow } 64 \text { threads to compete } \\
\text { for work, it is built on top of a lock-free queue as described by Michael and } \\
\text { Scott [1996]. It was applied to two square matrices both of dimension 1,024. }\end{array}$ \\
\hline SpanningTree & $\begin{array}{l}\text { An implementation of a parallel spanning tree algorithm built on top of a } \\
\text { work-stealing queue as described by Bader et al. [2005]. It was applied to a } \\
\text { graph of 10,000 nodes, each of degree 1,000. }\end{array}$ \\
\hline
\end{tabular}

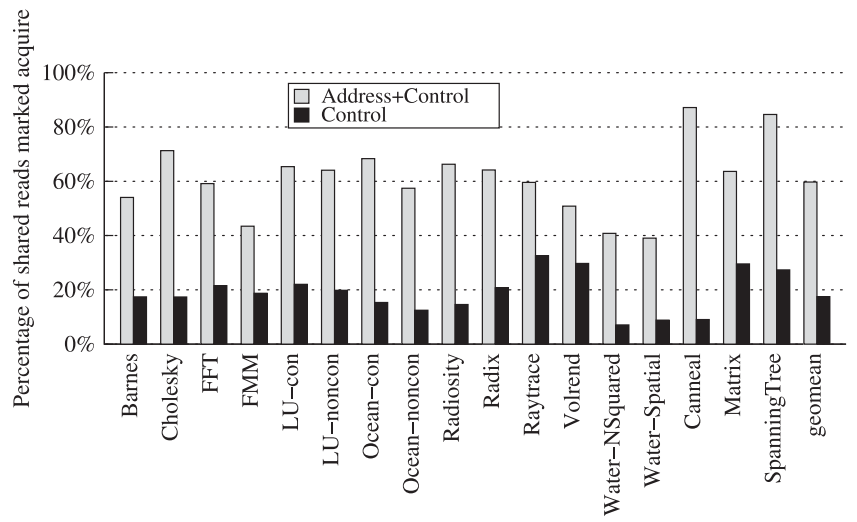

Fig. 7. Static percentage of potentially thread-escaping reads that our analysis marks as an acquire.

we present the reductions in the number of full memory fences placed for an x86-TSO machine, where only orderings of the form $w \rightarrow r$ require such enforcement. Finally, we present the performance improvements achieved over Pensieve. For the performance experiments, we used an Intel i3-2100 running Linux 3.2.0-67 (Ubuntu 12.04.4). All the programs were run using 64 threads.

\subsection{Synchronization Read Detection}

Applying the algorithms as defined in Section 4, we are able to mark a subset of the potentially escaping reads as acquires. The percentage of these reads that are marked acquires by each variant of our approach is presented as Figure 7.

As we can see, the Control acquires-only form of our analysis is able to greatly reduce the number of reads that must be treated as acquires. In the best case (WaterNSquared), only $7 \%$ are potentially acquires. On average, ${ }^{7}$ we see $18 \%$ of the reads marked as acquires. Even in the worst case, our analysis is able to significantly reduce the number of reads that must be treated as acquires. We see this in Raytrace, with $33 \%$ marked as acquires.

Using the Address+Control variant, we are still able to reduce the number of reads marked as acquires in all cases. On average, we see $60 \%$ marked as acquires. In the best case (Water-Spatial), only 39\% need be marked.

\subsection{Ordering Pruning}

Using the acquire detection results, we are able to prune the orderings considered by the fence placement algorithm. As detailed in Section 2.3, identifying acquires allows

\footnotetext{
${ }^{7}$ Geometric mean is used for all normalized results.
} 


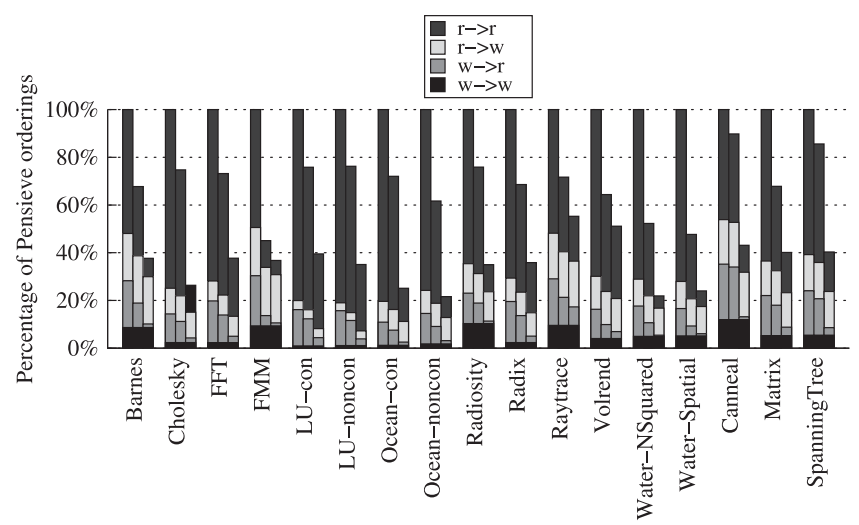

Fig. 8. A breakdown of orderings by type for Pensieve (left), Address+Control (center), and Control (right).

pruning of those $w \rightarrow r$ and $r \rightarrow r$ orderings that do not conform to the rules in Table I. Figure 8 presents the results of this pruning.

As Figure 8 shows, our Control approach significantly reduces the number of $w \rightarrow r$ and $r \rightarrow r$ orderings required to be considered for fence placement. This result holds across all the programs tested, with an average of 34\% orderings remaining after application of our approach. Because $r \rightarrow r$ orderings form the majority of orderings in all but two of the programs, reducing them has the largest overall impact on the number of orderings considered. $w \rightarrow r$ orderings are also pruned significantly, although because they often form only a small percentage of overall orderings, the impact of this on the total number of orderings is smaller. Because we do not identify a specific subset of writes as releases, $r \rightarrow w$ and $w \rightarrow w$ orderings are unaffected by the pruning process. With $w \rightarrow r$ and $r \rightarrow r$ orderings forming the majority of the orderings, the correlation between the percentage of reads marked as acquires (Figure 7) and the percentage of orderings that survive pruning is not unexpected.

Examining the results for the Address+Control variant, we see that reductions in $w \rightarrow r$ and $r \rightarrow r$ are still achieved. Specifically, only $68 \%$ orderings remain on average.

\subsection{Fence Placement}

In placing fences, we consider the requirements of an x86-TSO hardware model. Here, only $w \rightarrow r$ orderings require enforcement by a full memory fence. Other orderings are automatically enforced by the hardware and are enforced during the compilation process with empty memory-clobbering assembly instructions that have no presence in the final program. As Figure 8 showed, our pruning was very effective at reducing the number of $w \rightarrow r$ orderings.

Applying the fence minimization algorithm to the pruned sets of orderings for both variants of our approach and Pensieve for comparison, we determine the percentage of full fences that are still placed when using pruned orderings. This is shown as Figure 9.

As Figure 9 shows, the impact of pruning orderings is significant in reducing the static number of fences that the algorithm places to enforce $w \rightarrow r$ orderings. As we can see, the percentage of fences placed is quite strongly correlated with the percentage of reads marked as acquires (Figure 7). For the Control algorithm, we see on average 38\% of Pensieve's fences required, with Canneal receiving a $89 \%$ reduction in the number of fences placed. For the Address+Control variant, on average $73 \%$ of Pensieve's fences are required. 


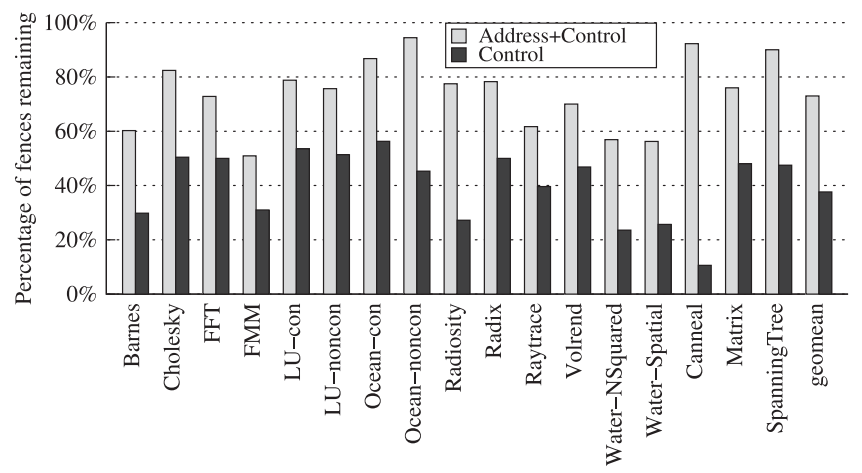

Fig. 9. Static percentage of full fences that remain on x86-TSO after using pruned orderings.

Despite the significant improvements that our pruning technique provides, it is not capable of eliminating all false positives on its own, and therefore some erroneous fences remain. Considering a relaxed machine, because all synchronization accesses must be identified to prevent compiler reordering, we determine manual fence placement. For the programs considered, we attempted to place as few fences as possible, and we believe we have achieved minimal fence placement. In Canneal, where the programmers have already identified fence positions for a variety of architectures, only 10 fences are required. In FMM, we require six fences to handle the ad hoc flag synchronizations. In Volrend, we require two fences to handle the ad hoc barrier implementation, despite the use of pthread locks. Matrix requires six fences, and, finally, in SpanningTree, we require five fences. The other programs are (to the best of our knowledge) well-synchronized by library calls to locks and barriers.

Even given the relatively small number of true synchronization accesses, expert manual fence placement is not a viable solution. This is due to the proliferation of ad hoc synchronizations inside programs with large code bases [Xiong et al. 2010] and the inability of race detectors to distinguish between synchronization and data accesses [Tian et al. 2008] such that both will be reported.

\subsection{Performance Improvements}

To examine the impact of reducing the number of fences, we executed the programs having applied Pensieve and both variants of our approach and normalize these against manual fence placement. Each of the experiments was repeated 100 times and averages taken. The results of these experiments are presented as Figure 10. We acknowledge that Pensieve guarantees SC in all cases, whereas our approach guarantees SC only for well-synchronized programs.

As we can see, in all cases, the fences placed using either variant of our approach results in a performance improvement over using a naive set of orderings. On average, we see that Pensieve is $1.94 \times$ slower than the baseline, with our Control approach being only $1.44 \times$ slower than the baseline. The Address+Control approach is $1.69 \times$ slower than the baseline. In other words, on average, our Control approach results in a $30 \%$ speedup over Pensieve, whereas the Address+Control approach results in executions $14 \%$ faster than Pensieve. In the best case (Matrix), we achieve a $90 \%$ improvement over Pensieve using Control. For the Address+Control approach, the best case (Water-Spatial) is $42 \%$ faster than Pensieve.

Examining the performance results for individual programs, we see that the speedups achieved over the naive are not strongly correlated with the changes in static 


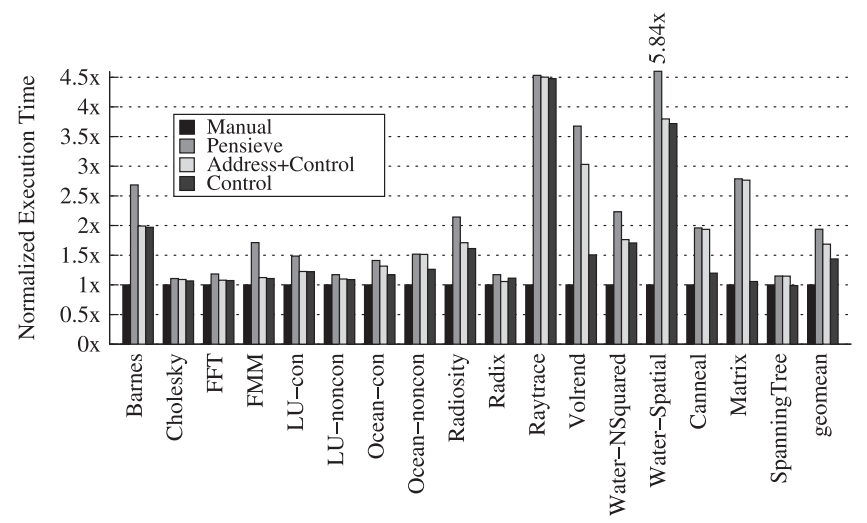

Fig. 10. Execution time with fences placed using Pensieve, Address+Control, and Control, normalized against manual fence placement.

fence placement. This is due to specific fences being reached more than others during the execution of the program. This is best highlighted by the case of Raytrace, where significant reductions in the number of static fences is not reflected in performance improvement. When looking at the results for Address+Control, we see that in some cases it is closer to Pensieve (e.g., Ocean-noncon) and in others (e.g., Water-Spatial) closer to Control. To which result Address+Control is most similar depends on the propensity of the use of escaping reads as addresses in heavily executed code regions. In one program (Radix), we see Address+Control outperforming the simple algorithm. This is likely due to the short running time and small number of fences placed, making the result susceptible to noise. This also accounts for why Control achieves a $1 \%$ improvement over the baseline for SpanningTree.

In terms of performance comparison with the manual baseline, we see that there is still some improvement possible. There are two reasons for this discrepancy. First is the difficult orthogonal problem of optimal fence minimization given a set of orderings to enforce. In extremis, this may even require profiling to determine the fence insertion points that have the minimal impact on performance. Second, although our signatures significantly prune the number of shared reads considered as acquires, some false positives still remain.

\section{RELATED WORK}

Programmer-centric memory models. Adve and Hill [1990] and Gharachorloo [1990] were the first to propose programmer-centric memory consistency models, where the system enforces SC as long as the programmer writes DRF programs and provides information about synchronization operations. Indeed, Adve's DRF-based models [Adve 1993] and Gharachorloo's Properly Labeled-based models [Gharachorloo 1995] are the precursors to the memory consistency models adopted by languages such as C [Boehm and Adve 2008] and Java [Manson et al. 2005]. The main difference between these works and ours is that, whereas they assume programmer-annotated synchronization labels, we assume unlabeled DRF programs.

Delay-set analysis. Shasha and Snir [1988] were the first to consider the problem of computing the minimum number of memory orderings (delays) to ensure that a concurrent shared memory program satisfies SC. In this work, we focus on how the 
given orderings can be pruned if the shared memory program is a DRF (but unlabeled) program. To put it succinctly, we do Delay-set analysis for unlabeled DRF programs.

A more recent work [Alglave et al. 2014] attempts to address the scalability issues inherent in Delay-set analysis by examining an overapproximation of the critical cycles. It is, however, limited in failing to handle recursion and dynamic thread creation, the latter of which is common in the programs examined in our evaluation. Specifically, this tool does not handle pthread_create calls in loops that could not be statically unrolled. We note, however, that our signatures would be equally applicable to those in Alglave et al. [2014], and our choice to build on top of Pensieve is due to its lack of the limitations described earlier.

Fence minimization. There have been a number of works [Fang et al. 2003; Kamil et al. 2005; Wong et al. 2002] that focus on computing the minimal number of fences for satisfying the orderings given by Delay-set analysis. These works are orthogonal to our work because these can very well be applied for satisfying the pruned orderings given by our analysis.

Synchronization detection. Our work is related to prior work [Tian et al. 2008, 2009; Xiong et al. 2010] on busy-wait synchronization detection. Tian et al. [2008, 2009] proposed a dynamic analysis technique for identifying user-defined busy-wait synchronizations. Because that work uses dynamic analysis, they suffer from false negatives: In other words, some synchronizations can be missed. Subsequently, Xiong et al. [2010] showed how synchronizations can be identified using static analysis, so that there can be no false negatives. Our work differs from theirs in one important aspect: Their analysis is only applicable for busy-wait synchronization; thus, it will miss identifying acquires used in nonblocking algorithms, such as those used in our evaluation. It is worth noting that missing such acquires leads to correctness issues in our context, which explains why the mentioned detectors cannot be used in the context of our work. Indeed, one of the nice side effects of our work is that, to the best of our knowledge, ours is the first general acquire detector.

Hardware-based memory ordering. There have been a number of recent works [Blundell et al. 2009; Gharachorloo et al. 1991; Gniady et al. 1999; Lin et al. 2010; Singh et al. 2012] that have proposed techniques for efficiently enforcing memory ordering. In contrast with these works, each of which involve hardware support, we do not use any hardware support. Furthermore, each of the listed works are orthogonal to us, in that they can very well be used to efficiently enforce the pruned orderings given by our work.

SC-preserving compiler. Ahn et al. [2009] proposed the Bulk compiler, which together with Bulk hardware (which enforces hardware SC at chunk level) guarantees SC at the language level. In other words, the Bulk compiler preserves SC by ensuring that it does not reorder memory operations across chunks. More recently, Marino et al. [2011] proposed the SC-preserving compiler, which together with SC hardware (which enforces $\mathrm{SC}$ at the hardware level) guarantees SC at the language level. Their main result is that it is possible for the compiler to preserve $\mathrm{SC}$ without significant slowdown $(<5 \%$ on average across a suite of parallel programs). On the other hand, they assume that the hardware cannot reorder operations (i.e., they assume that the hardware enforces SC). In contrast, our work considers the problem of how to enforce SC on hardware that could reorder memory operations. Of course, to preserve SC at the language level , we would need a compiler that preserves SC (i.e., the just listed works). Recall that in our implementation we ensure that the compiler cannot reorder shared memory operations by inserting an empty memory-clobbering assembly instruction between such operations, which LLVM interprets as a compiler fence. It is worth noting that 
this corresponds to the naive-SC variant [Marino et al. 2011]. We could have very well used the SC-preserving compiler proposed (with all optimizations), which could potentially translate into better performance. In this respect, our work is orthogonal to the mentioned works.

\section{CONCLUSION}

Relaxed hardware memory consistency models are used to ensure performance in multicore computers. A large body of legacy code assumes SC. Placing sufficient but minimal fences is challenging. The starting point of understanding the required placement is Delay-set analysis. However, in practice, approximations are applied, resulting in many superfluous orderings.

With Delay-set analysis too hard in the general case and with languages converging to DRF-based memory models, we, for the first time, attack the problem of Delay-set analysis for legacy DRF programs. We prove that a read of shared data must match at least one of two signatures to be an acquire. We determine that this enables the pruning of a large number of orderings, thus reducing the set that need be considered for fence placement.

Developing both simple (control acquires) and conservative (control and address acquires) algorithms, we implement them in LLVM and demonstrate the significance of our contribution. Applying our control acquire detection on a set of lock-free programs and to SPLASH-2, we reduce the average number of orderings considered by $66 \%$. Using a fence minimization technique, this translates to an average of $62 \%$ fewer fences on $\mathrm{x} 86$-TSO and up to $2.64 \times$ speedup over an existing practical technique.

\section{ACKNOWLEDGMENTS}

We thank the anonymous reviewers for their helpful comments for improving this article. This research is supported by EPSRC grant EP/L000725/1 and an Intel early career faculty award to the University of Edinburgh.

\section{REFERENCES}

Sarita Vikram Adve. 1993. Designing memory consistency models for shared-memory multiprocessors. Ph.D. Dissertation. University of Wisconsin, Madison, WI, USA.

Sarita V. Adve and Kourosh Gharachorloo. 1995. Shared memory consistency models: A tutorial. IEEE Computer 29 (1995), 66-76.

Sarita V. Adve and Mark D. Hill. 1990. Weak ordering-A new definition. In Proceedings of the 17th Annual International Symposium on Computer Architecture (ISCA'90). ACM, New York, NY, USA, 2-14.

W. Ahn, S. Qi, M. Nicolaides, J. Torrellas, J.-W. Lee, X. Fang, S. Midkiff, and David Wong. 2009. BulkCompiler: High-performance sequential consistency through cooperative compiler and hardware support. In Proceedings of the 42nd Annual IEEE/ACM International Symposium on Microarchitecture (MICRO'42). ACM, New York, NY, USA, 133-144.

Jade Alglave, Daniel Kroening, Vincent Nimal, and Daniel Poetzl. 2014. Don't sit on the fence-A static analysis approach to automatic fence insertion. In Proceedings of the 26th International Conference on Computer Aided Verification (CAV'14), Held as Part of the Vienna Summer of Logic, VSL 2014. Vienna, Austria, 508-524.

David A. Bader and Guojing Cong. 2005. A fast, parallel spanning tree algorithm for symmetric multiprocessors (SMPs). Journal of Parallel Distributed Computing 65, 9 (2005), 994-1006.

Mark Batty, Scott Owens, Susmit Sarkar, Peter Sewell, and Tjark Weber. 2011. Mathematizing C++ concurrency. In Proceedings of the 38th Annual ACM SIGPLAN-SIGACT Symposium on Principles of Programming Languages (POPL'11). ACM, New York, NY, USA, 55-66.

Christian Bienia, Sanjeev Kumar, Jaswinder Pal Singh, and Kai Li. 2008. The PARSEC benchmark suite: Characterization and architectural implications. In Proceedings of the 17th International Conference on Parallel Architectures and Compilation Techniques (PACT'08). ACM, New York, NY, USA, 72-81. 
Colin Blundell, Milo M. K. Martin, and Thomas F. Wenisch. 2009. InvisiFence: Performance-transparent memory ordering in conventional multiprocessors. In Proceedings of the 36th Annual International Symposium on Computer Architecture (ISCA'09). ACM, New York, NY, USA, 233-244.

Hans-Juergen Boehm and Sarita V. Adve. 2008. Foundations of the C++ concurrency memory model. In Proceedings of the 29th ACM SIGPLAN Conference on Programming Language Design and Implementation (PLDI'08). ACM, New York, NY, USA, 68-78.

Luis Ceze, James Tuck, Pablo Montesinos, and Josep Torrellas. 2007. BulkSC: Bulk enforcement of sequential consistency. SIGARCH Computer Architecture News 35, 2 (2007), 278-289.

David Chase and Yossi Lev. 2005. Dynamic circular work-stealing deque. In Proceedings of the 17th Annual ACM Symposium on Parallelism in Algorithms and Architectures (SPAA'05). ACM, New York, NY, USA, 21-28.

Daniel Chazan and Willard Miranker. 1969. Chaotic relaxation. Linear Algebra and Its Applications 2, 2 (1969), 199-222.

Travis Craig. 1994. Building FIFO and Priority-Queuing Spin Locks from Atomic Swap. Technical Report. Technical Report 93-02-02, University of Washington, Seattle, Washington.

Tudor David, Rachid Guerraoui, and Vasileios Trigonakis. 2013. Everything you always wanted to know about synchronization but were afraid to ask. In ACM SIGOPS 24th Symposium on Operating Systems Principles (SOSP'13). Farmington, PA, USA, 33-48.

E. W. Dijkstra. 1965. Solution of a problem in concurrent programming control. Communications of the ACM 8, 9 (Sept. 1965), 569.

Xing Fang, Jaejin Lee, and Samuel P. Midkiff. 2003. Automatic fence insertion for shared memory multiprocessing. In Proceedings of the 17th Annual International Conference on Supercomputing (ICS'03). ACM, New York, NY, USA, 285-294.

Matteo Frigo, Charles E. Leiserson, and Keith H. Randall. 1998. The implementation of the Cilk-5 multithreaded language. SIGPLAN Notes 33, 5 (May 1998), 212-223.

Andreas Frommer and Daniel B Szyld. 2000. On asynchronous iterations. Journal of Computational and Applied Mathematics 123, 1 (2000), 201-216.

Kourosh Gharachorloo. 1995. Memory Consistency Models for Shared-Memory Multiprocessors. Ph.D. Dissertation. Stanford University.

Kourosh Gharachorloo, Anoop Gupta, and John L. Hennessy. 1991. Two techniques to enhance the performance of memory consistency models. In Proceedings of the 1991 International Conference on Parallel Processing (ICPP'91). 355-364.

Kourosh Gharachorloo, Daniel Lenoski, James Laudon, Phillip Gibbons, Anoop Gupta, and John Hennessy. 1990. Memory consistency and event ordering in scalable shared-memory multiprocessors. In Proceedings of the 17th Annual International Symposium on Computer Architecture (ISCA'90). ACM, New York, NY, USA, 15-26.

Chris Gniady, Babak Falsafi, and T. N. Vijaykumar. 1999. Is SC + ILP=RC?. In Proceedings of the Twenty Sixth Annual International Symposium on Computer Architecture (ISCA'99). IEEE Computer Society, Washington, DC, USA, 162-171.

Mark D. Hill. 1998. Multiprocessors should support simple memory-consistency models. Computer 31, 8 (1998), 28-34.

Amir Kamil, Jimmy Su, and Katherine Yelick. 2005. Making sequential consistency practical in titanium. In Proceedings of the 2005 ACM / IEEE Conference on Supercomputing (SC'05). IEEE Computer Society, Washington, DC, USA, 15.

L. Lamport. 1979. How to make a multiprocessor computer that correctly executes multiprocess program. IEEE Transactions on Computing 28, 9 (1979), 690-691.

Leslie Lamport. 1987. A fast mutual exclusion algorithm. ACM Transactions on Computer Systems 5, 1 (Jan. 1987), 1-11.

Jaejin Lee and David A. Padua. 2001. Hiding relaxed memory consistency with a compiler. IEEE Transactions on Computing 50, 8 (2001), 824-833.

Changhui Lin, Vijay Nagarajan, and Rajiv Gupta. 2010. Efficient sequential consistency using conditional fences. In Proceedings of the 19th International Conference on Parallel Architectures and Compilation Techniques (PACT'10). ACM, 295-306.

Jeremy Manson, William Pugh, and Sarita V. Adve. 2005. The Java memory model. In Proceedings of the 32 d ACM SIGPLAN-SIGACT Symposium on Principles of Programming Languages (POPL'O5). ACM, New York, NY, USA, 378-391.

Daniel Marino, Abhayendra Singh, Todd D. Millstein, Madanlal Musuvathi, and Satish Narayanasamy. 2011. A case for an SC-preserving compiler. In Proceedings of the 32nd ACM SIGPLAN Conference on Programming Language Design and Implementation (PLDI'11). ACM, New York, NY, USA, 199-210. 
Andrew J. McPherson, Vijay Nagarajan, Susmit Sarkar, and Marcelo Cintra. 2015. Fence placement for legacy data-race-free programs via synchronization read detection. In Proceedings of the 20th ACM SIGPLAN Symposium on Principles and Practice of Parallel Programming (PPoPP'15). ACM, New York, NY, USA, 249-250.

John M. Mellor-Crummey and Michael L. Scott. 1991. Algorithms for scalable synchronization on sharedmemory multiprocessors. ACM Transactions on Computer Systems 9, 1 (Feb. 1991), 21-65.

Maged M. Michael and Michael L. Scott. 1996. Simple, fast, and practical non-blocking and blocking concurrent queue algorithms. In Proceedings of the Fifteenth Annual ACM Symposium on Principles of Distributed Computing (PODC'96). ACM, New York, NY, USA, 267-275.

Adrian Nistor, Darko Marinov, and Josep Torrellas. 2010. InstantCheck: Checking the determinism of parallel programs using on-the-fly incremental hashing. In 43rd Annual IEEE/ACM International Symposium on Microarchitecture (MICRO'10). Atlanta, Georgia, USA, 251-262.

Gary L. Peterson. 1981. Myths about the mutual exclusion problem. Information Processing Letter 12, 3 (1981), 115-116.

Dennis Shasha and Marc Snir. 1988. Efficient and correct execution of parallel programs that share memory. ACM Transactions on Programming Languages and Systems 10, 2 (1988), 282-312.

Abhayendra Singh, Satish Narayanasamy, Daniel Marino, Todd D. Millstein, and Madanlal Musuvathi. 2012. End-to-end sequential consistency. In Proceedings of the 39th Annual International Symposium on Computer Architecture (ISCA'12). 524-535.

Zehra Sura, Xing Fang, Chi-Leung Wong, Samuel P. Midkiff, Jaejin Lee, and David Padua. 2005. Compiler techniques for high performance sequentially consistent Java programs. In Proceedings of the Tenth ACM SIGPLAN Symposium on Principles and Practice of Parallel Programming (PPoPP'05). ACM, New York, NY, USA, 2-13.

B. K. Szymanski. 1988. A simple solution to lamport's concurrent programming problem with linear wait. In Proceedings of the 2nd International Conference on Supercomputing (ICS'88). ACM, New York, NY, USA, 621-626.

Chen Tian, Vijay Nagarajan, Rajiv Gupta, and Sriraman Tallam. 2008. Dynamic recognition of synchronization operations for improved data race detection. In Proceedings of the 2008 International Symposium on Software Testing and Analysis (ISSTA'08). ACM, New York, NY, USA, 143-154.

Chen Tian, Vijay Nagarajan, Rajiv Gupta, and Sriraman Tallam. 2009. Automated dynamic detection of busy-wait synchronizations. Software: Practice and Experience 39, 11 (2009), 947-972.

Chi-Leung Wong, Zehra Sura, David A. Padua, Xing Fang, Jaejin Lee, and Samuel P. Midkiff. 2002. The pensieve project: A compiler infrastructure for memory models. In International Symposium on Parallel Architectures, Algorithms and Networks (ISPAN'02). Makati City, Metro Manila, Philippines, 239-244.

Steven Cameron Woo, Moriyoshi Ohara, Evan Torrie, Jaswinder Pal Singh, and Anoop Gupta. 1995. The SPLASH-2 programs: Characterization and methodological considerations. In Proceedings of the 22nd Annual International Symposium on Computer Architecture (ISCA'95). ACM, New York, NY, USA, 24-36.

Weiwei Xiong, Soyeon Park, Jiaqi Zhang, Yuanyuan Zhou, and Zhiqiang Ma. 2010. Ad hoc synchronization considered harmful. In Proceedings of the 9th USENIX Conference on Operating Systems Design and Implementation (OSDI'10). USENIX Association, Berkeley, CA, USA, 1-8.

Received January 2015; revised August 2015; accepted October 2015 